\title{
Weight bases of Gelfand-Tsetlin type for representations of classical Lie algebras
}

\author{
A. I. MOLEV \\ School of Mathematics and Statistics \\ University of Sydney, NSW 2006, Australia \\ alexm@maths.usyd.edu.au
}

\begin{abstract}
This paper completes a series devoted to explicit constructions of finitedimensional irreducible representations of the classical Lie algebras. Here the case of odd orthogonal Lie algebras (of type $B$ ) is considered (two previous papers dealt with $C$ and $D$ types). A weight basis for each representation of the Lie algebra $\mathfrak{o}(2 n+1)$ is constructed. The basis vectors are parametrized by Gelfand-Tsetlin-type patterns. Explicit formulas for the matrix elements of generators of $\mathfrak{o}(2 n+1)$ in this basis are given. The construction is based on the representation theory of the Yangians. A similar approach is applied to the $A$ type case where the well-known formulas due to Gelfand and Tsetlin are reproduced.
\end{abstract}




\section{Introduction}

In this paper we give an explicit construction of each finite-dimensional irreducible representation $V$ of an odd orthogonal Lie algebra $\mathfrak{o}(2 n+1)$ (i.e. a simple complex Lie algebra of type $B$ ). A weight basis in $V$ is obtained by the application of certain elements of the enveloping algebra (the lowering operators) to the highest weight vector. Explicit formulas for the matrix elements of generators of the Lie algebra $\mathfrak{o}(2 n+1)$ in this basis are given. We follow an approach applied in the previous papers [13] and [14] where similar results were obtained for the $C$ and $D$ type Lie algebras. We also reproduce a slightly modified version of the well-known construction of the Gelfand-Tsetlin bases for the $A$ type Lie algebras.

Let $\mathfrak{g}_{n}$ denote the rank $n$ simple Lie algebra of type $A, B, C$, or $D$. The restriction of a finite-dimensional irreducible representation $V$ of $\mathfrak{g}_{n}$ to the subalgebra $\mathfrak{g}_{n-1}$ is multiplicity-free for the $A$ type case, and it is not necessarily so for the $B, C, D$ types. Gelfand and Tsetlin [5] used the chain of subalgebras

$$
\mathfrak{g}_{1} \subset \mathfrak{g}_{2} \subset \cdots \subset \mathfrak{g}_{n}
$$

to parametrize basis vectors in $V$ and give formulas for the matrix elements of generators for the $A$ type case. Different approaches to derive these formulas are used e.g. in [24, 17, 25, 7, 18, 11].

Analogous results for representations of the orthogonal Lie algebra are obtained by Gelfand and Tsetlin in [6]; see also [21, 23, 8]. Here the chain (1.1) is replaced with the one which involves both the odd and even orthogonal Lie algebras. However, the corresponding basis vectors lose the weight property, i.e. they are not eigenvectors for the elements of the Cartan subalgebra. To get a weight basis we propose to use the chain (1.1) for the $B, C, D$ types as well. We "separate" the multiplicities occurring in the reduction $\mathfrak{g}_{n} \downarrow \mathfrak{g}_{n-1}$ by applying the representation theory of the Yangians. Namely, the subspace $V_{\mu}^{+}$of $\mathfrak{g}_{n-1}$-highest vectors of weight $\mu$ in $V$ possesses a natural structure of a representation of the twisted Yangian $\mathrm{Y}^{+}(2)$ or $\mathrm{Y}^{-}(2)$, in the orthogonal and symplectic case, respectively. The twisted Yangians are introduced and studied by Olshanski [20]; see also [16] for a detailed exposition. The action of $\mathrm{Y}^{ \pm}(2)$ in the space $V_{\mu}^{+}$arises from his centralizer construction [20]. Finite-dimensional irreducible representations of the twisted Yangians are classified in [12]. In particular, it turns out that the representation $V_{\mu}^{+}$of $\mathrm{Y}^{ \pm}(2)$ can be extended to a larger algebra, the Yangian $\mathrm{Y}(2)$ for the Lie algebra $\mathfrak{g l}(2)$. The algebra $\mathrm{Y}(2)$ and its representations are very well studied; see [22], 2]. In particular, a large class of representation of $\mathrm{Y}(2)$ 
admits Gelfand-Tsetlin-type bases associated with the inclusion $\mathrm{Y}(1) \subset \mathrm{Y}(2)$; see [11, 19]. This allows us to get a natural basis in the space $V_{\mu}^{+}$, and then by induction to get a basis in the entire space $V$.

Note that in the case of $C$ or $D$ type the $\mathrm{Y}(2)$-module $V_{\mu}^{+}$is irreducible while in the $B$ type case it is a direct sum of two irreducible submodules. This does not lead, however, to major differences in the constructions, and the final formulas are similar in all the three cases.

Our calculations of the matrix elements of the generators of $\mathfrak{g}_{n}$ are based on the relationship between the twisted Yangian $\mathrm{Y}^{ \pm}(2)$ and the transvector algebra $\mathrm{Z}\left(\mathfrak{g}_{n}, \mathfrak{g}_{n-1}\right)$ (it is also called the Mickelsson algebra or S-algebra). It is generated by the raising and lowering operators which preserve the subspace $V^{+}$of $\mathfrak{g}_{n-1}$-highest vectors in $V$. The algebraic structure of the transvector algebras is studied in detail in [26] with the use of the extremal projections for reductive Lie algebras [1]. We construct an algebra homomorphism $\mathrm{Y}^{ \pm}(2) \rightarrow \mathrm{Z}\left(\mathfrak{g}_{n}, \mathfrak{g}_{n-1}\right)$ which allows us to express the generators of the twisted Yangian, as operators in $V_{\mu}^{+}$, in terms of the raising and lowering operators. This plays a key role in the calculation of the matrix elements of the generators of $\mathfrak{g}_{n}$ in the basis of $V$.

Explicit combinatorial constructions of the fundamental representations of the symplectic and odd orthogonal Lie algebras were recently given by Donnelly [3]. He also showed that in the symplectic case the basis of [13] for the fundamental representations coincides, up to a scaling, with a basis of his «1. It is likely that a similar connection exists in the odd orthogonal case.

\section{Gelfand-Tsetlin basis for $\mathfrak{g l}(n)$}

Let $E_{i j}, i, j=1, \ldots, n$ denote the standard basis of the general linear Lie algebra $\mathfrak{g}_{n}=\mathfrak{g l}(n)$ over the field of complex numbers. The subalgebra $\mathfrak{g}_{n-1}$ is spanned by the basis elements $E_{i j}$ with $i, j=1, \ldots, n-1$. Denote by $\mathfrak{h}=\mathfrak{h}_{n}$ the diagonal Cartan subalgebra in $\mathfrak{g}_{n}$. The elements $E_{11}, \ldots, E_{n n}$ form a basis of $\mathfrak{h}$.

Finite-dimensional irreducible representations of $\mathfrak{g}_{n}$ are in a one-to-one correspondence with $n$-tuples of complex numbers $\lambda=\left(\lambda_{1}, \ldots, \lambda_{n}\right)$ such that

$$
\lambda_{i}-\lambda_{i+1} \in \mathbb{Z}_{+} \quad \text { for } \quad i=1, \ldots, n-1 .
$$

Such an $n$-tuple $\lambda$ is called the highest weight of the corresponding representation which we shall denote by $L(\lambda)$. It contains a unique, up to a multiple, nonzero 
vector $\xi$ (the highest vector) such that $E_{i i} \xi=\lambda_{i} \xi$ for $i=1, \ldots, n$ and $E_{i j} \xi=0$ for $1 \leq i<j \leq n$. Denote by $L(\lambda)^{+}$the subspace of $\mathfrak{g}_{n-1}$-highest vectors in $L(\lambda)$ :

$$
L(\lambda)^{+}=\left\{\eta \in L(\lambda) \mid E_{i j} \eta=0, \quad 1 \leq i<j<n\right\}
$$

Given a $\mathfrak{g}_{n-1}$-highest weight $\mu=\left(\mu_{1}, \ldots, \mu_{n-1}\right)$ we denote by $L(\lambda)_{\mu}^{+}$the corresponding weight subspace in $L(\lambda)^{+}$:

$$
L(\lambda)_{\mu}^{+}=\left\{\eta \in L(\lambda)^{+} \mid E_{i i} \eta=\mu_{i} \eta, \quad i=1, \ldots, n-1\right\} .
$$

It is well-known 24 that the space $L(\lambda)_{\mu}^{+}$is either trivial or one-dimensional. Moreover, $\operatorname{dim} L(\lambda)_{\mu}^{+}=1$ if and only if

$$
\lambda_{i}-\mu_{i} \in \mathbb{Z}_{+} \quad \text { and } \quad \mu_{i}-\lambda_{i+1} \in \mathbb{Z}_{+} \quad \text { for } \quad i=1, \ldots, n-1 .
$$

In other words, the restriction of $L(\lambda)$ to the subalgebra $\mathfrak{g}_{n-1}$ is multiplicity-free:

$$
\left.L(\lambda)\right|_{\mathfrak{g}_{n-1}} \simeq \bigoplus L^{\prime}(\mu)
$$

where $L^{\prime}(\mu)$ is the irreducible $\mathfrak{g}_{n-1}$-module with the highest weight $\mu$ satisfying the conditions (2.1). A parameterization of basis vectors in $L(\lambda)$ is obtained by using its further restrictions to the subalgebras of the chain (1.1). A Gelfand-Tsetlin pattern $\Lambda$ associated with $\lambda$ is an array of row vectors

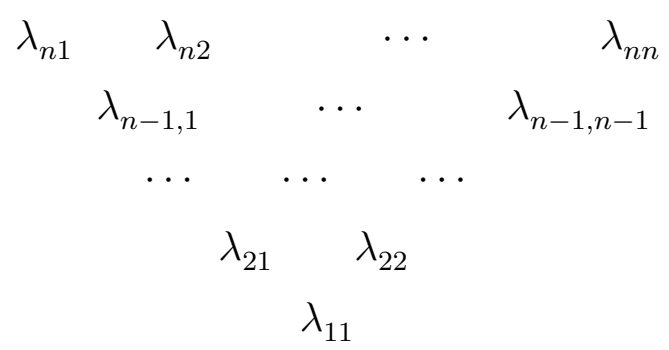

such that the upper row coincides with $\lambda$ and the following conditions hold

$$
\lambda_{k i}-\lambda_{k-1, i} \in \mathbb{Z}_{+}, \quad \lambda_{k-1, i}-\lambda_{k, i+1} \in \mathbb{Z}_{+}, \quad i=1, \ldots, k-1
$$

for each $k=2, \ldots, n$.

Remark. If the highest weight $\lambda$ is a partition then there is a natural bijection between the patterns associated with $\lambda$ and semistandard $\lambda$-tableaux with entries in $\{1, \ldots, n\}$. A pattern can be viewed as a sequence of partitions

$$
\lambda^{(1)} \subseteq \lambda^{(2)} \subseteq \cdots \subseteq \lambda^{(n)}=\lambda,
$$


with $\lambda^{(k)}=\left(\lambda_{k 1}, \ldots, \lambda_{k k}\right)$. Conditions (2.2) mean that the skew diagram $\lambda^{(k)} / \lambda^{(k-1)}$ is a horizontal strip; see e.g. [10].

Let us set $l_{k i}=\lambda_{k i}-i+1$.

Theorem 2.1 There exists a basis $\left\{\xi_{\Lambda}\right\}$ in $L(\lambda)$ parametrized by all patterns $\Lambda$ such that the action of generators of $\mathfrak{g}_{n}$ is given by the formulas

$$
\begin{aligned}
E_{k k} \xi_{\Lambda} & =\left(\sum_{i=1}^{k} \lambda_{k i}-\sum_{i=1}^{k-1} \lambda_{k-1, i}\right) \xi_{\Lambda}, \\
E_{k, k+1} \xi_{\Lambda} & =-\sum_{i=1}^{k} \frac{\left(l_{k i}-l_{k+1,1}\right) \cdots\left(l_{k i}-l_{k+1, k+1}\right)}{\left(l_{k i}-l_{k 1}\right) \cdots \wedge \cdots\left(l_{k i}-l_{k k}\right)} \xi_{\Lambda+\delta_{k i}}, \\
E_{k+1, k} \xi_{\Lambda} & =\sum_{i=1}^{k} \frac{\left(l_{k i}-l_{k-1,1}\right) \cdots\left(l_{k i}-l_{k-1, k-1}\right)}{\left(l_{k i}-l_{k 1}\right) \cdots \wedge \cdots\left(l_{k i}-l_{k k}\right)} \xi_{\Lambda-\delta_{k i}} .
\end{aligned}
$$

The arrays $\Lambda \pm \delta_{k i}$ are obtained from $\Lambda$ by replacing $\lambda_{k i}$ by $\lambda_{k i} \pm 1$. It is supposed that $\xi_{\Lambda}=0$ if the array $\Lambda$ is not a pattern; the symbol $\wedge$ indicates that the zero factor in the denominator is skipped.

Proof. Consider the extension of the universal enveloping algebra $\mathrm{U}\left(\mathfrak{g}_{n}\right)$

$$
\mathrm{U}^{\prime}\left(\mathfrak{g}_{n}\right)=\mathrm{U}\left(\mathfrak{g}_{n}\right) \otimes_{\mathrm{U}(\mathfrak{h})} \mathrm{R}(\mathfrak{h})
$$

where $R(\mathfrak{h})$ is the field of fractions of the commutative algebra $U(\mathfrak{h})$. Let $J$ denote the left ideal in $\mathrm{U}^{\prime}\left(\mathfrak{g}_{n}\right)$ generated by the elements $E_{i j}$ with $1 \leq i<j<n$. Introduce the normalizer of $\mathrm{J}$ in $\mathrm{U}^{\prime}\left(\mathfrak{g}_{n}\right)$ :

$$
\text { Norm } \mathrm{J}=\left\{x \in \mathrm{U}^{\prime}\left(\mathfrak{g}_{n}\right) \mid \mathrm{J} x \subseteq \mathrm{J}\right\} .
$$

Note that $\mathrm{J}$ is a two-sided ideal in the algebra Norm J. We define the transvector algebra $\mathrm{Z}\left(\mathfrak{g}_{n}, \mathfrak{g}_{n-1}\right)$ as the quotient

$$
\mathrm{Z}\left(\mathfrak{g}_{n}, \mathfrak{g}_{n-1}\right)=\operatorname{Norm} \mathrm{J} / \mathrm{J}
$$

see [25, 26]. Equivalently, $\mathrm{Z}\left(\mathfrak{g}_{n}, \mathfrak{g}_{n-1}\right)$ can be defined by using the extremal projection $p=p_{n-1}$ for the Lie algebra $\mathfrak{g}_{n-1}$ [1]. The projection $p$ is, up to a factor from $\mathrm{R}\left(\mathfrak{h}_{n-1}\right)$, a unique element of an extension of $\mathrm{U}^{\prime}\left(\mathfrak{g}_{n-1}\right)$ to an algebra of formal series, satisfying the conditions

$$
E_{i j} p=p E_{j i}=0 \quad \text { for } \quad 1 \leq i<j \leq n-1 .
$$


The element $p$ is of zero weight (with respect to the adjoint action of $\mathfrak{h}_{n-1}$ ) and it can be normalized to satisfy the condition $p^{2}=p$. The projection $p$ is a welldefined operator in the quotient $\mathrm{U}^{\prime}\left(\mathfrak{g}_{n}\right) / \mathrm{J}$ which allows one to naturally identify the transvector algebra $\mathrm{Z}\left(\mathfrak{g}_{n}, \mathfrak{g}_{n-1}\right)$ with the image of $p$ [26]:

$$
\mathrm{Z}\left(\mathfrak{g}_{n}, \mathfrak{g}_{n-1}\right)=p\left(\mathrm{U}^{\prime}\left(\mathfrak{g}_{n}\right) / \mathrm{J}\right)
$$

An analog of the Poincaré-Birkhoff-Witt theorem holds for the algebra $\mathrm{Z}\left(\mathfrak{g}_{n}, \mathfrak{g}_{n-1}\right)$ so that ordered monomials in the elements $p E_{i n}$ and $p E_{n i}$ with $i=1, \ldots, n-1$ form a basis of $\mathrm{Z}\left(\mathfrak{g}_{n}, \mathfrak{g}_{n-1}\right)$ as a left or right $\mathrm{R}(\mathfrak{h})$-module [26]. These elements are called the raising and lowering operators and can be given by

$$
\begin{aligned}
p E_{i n} & =\sum_{i>i_{1}>\cdots>i_{s} \geq 1} E_{i i_{1}} E_{i_{1} i_{2}} \cdots E_{i_{s-1} i_{s}} E_{i_{s} n} \frac{1}{\left(h_{i}-h_{i_{1}}\right) \cdots\left(h_{i}-h_{i_{s}}\right)}, \\
p E_{n i} & =\sum_{i<i_{1}<\cdots<i_{s}<n} E_{i_{1} i} E_{i_{2} i_{1}} \cdots E_{i_{s} i_{s-1}} E_{n i_{s}} \frac{1}{\left(h_{i}-h_{i_{1}}\right) \cdots\left(h_{i}-h_{i_{s}}\right)},
\end{aligned}
$$

where $s=0,1, \ldots$ and $h_{i}=E_{i i}-i+1$. We shall also use normalized operators defined by

$$
\begin{aligned}
& z_{i n}=p E_{i n}\left(h_{i}-h_{i-1}\right) \cdots\left(h_{i}-h_{1}\right) \\
& z_{n i}=p E_{n i}\left(h_{i}-h_{i+1}\right) \cdots\left(h_{i}-h_{n-1}\right)
\end{aligned}
$$

These can be viewed as elements of the enveloping algebra $\mathrm{U}\left(\mathfrak{g}_{n}\right)$; cf. [24, 17]. We have the following relations [25, 26]

$$
\begin{array}{ll}
z_{n i} z_{n j}=z_{n j} z_{n i} & \text { for all } i, j, \\
z_{i n} z_{n j}=z_{n j} z_{i n} & \text { for } \quad i \neq j .
\end{array}
$$

Indeed, assume that $i<j$. Then (2.6) and (2.7) imply that in $\mathrm{Z}\left(\mathfrak{g}_{n}, \mathfrak{g}_{n-1}\right)$

$$
p E_{n i} p E_{n j}=p E_{n i} E_{n j}, \quad p E_{n j} p E_{n i}=p E_{n i} E_{n j} \frac{h_{i}-h_{j}+1}{h_{i}-h_{j}} .
$$

Now (2.9) follows from (2.8). The proof of (2.10) is similar.

Due to (2.6) the operators $z_{i n}$ and $z_{n i}$ preserve the space $L(\lambda)^{+}$: for $i=1, \ldots, n-1$

$$
z_{i n}: L(\lambda)_{\mu}^{+} \rightarrow L(\lambda)_{\mu+\delta_{i}}^{+}, \quad z_{n i}: L(\lambda)_{\mu}^{+} \rightarrow L(\lambda)_{\mu-\delta_{i}}^{+}
$$

where $\mu \pm \delta_{i}$ is obtained from $\mu$ by replacing $\mu_{i}$ with $\mu_{i} \pm 1$.

The following is a key lemma in the derivation of the Gelfand-Tsetlin formulas. 
Lemma 2.2 Given $\mu$ satisfying (2.1) the vector

$$
\xi_{\mu}=z_{n 1}^{\lambda_{1}-\mu_{1}} \cdots z_{n, n-1}^{\lambda_{n-1}-\mu_{n-1}} \xi
$$

spans the subspace $L(\lambda)_{\mu}^{+}$and for each $i=1, \ldots, n-1$ we have

$$
z_{i n} \xi_{\mu}=-\left(m_{i}-l_{1}\right) \cdots\left(m_{i}-l_{n}\right) \xi_{\mu+\delta_{i}}
$$

where $m_{i}=\mu_{i}-i+1, l_{i}=\lambda_{i}-i+1$. It is supposed that $\xi_{\mu+\delta_{i}}=0$ if $\lambda_{i}=\mu_{i}$.

Proof. By (2.11) the vector $\xi_{\mu}$ belongs to the subspace $L(\lambda)_{\mu}^{+}$. We need to show that it is nonzero. This will follow from relations (2.12). We shall outline a proof of these relations which involves the use of the Yangians; cf. [25]. Consider the $n \times n$-matrix $E$ whose $i j$-th entry is $E_{i j}$ and let $u$ be a formal variable. Introduce the polynomial $T(u)$ with coefficients in the universal enveloping algebra $\mathrm{U}\left(\mathfrak{g}_{n}\right)$ :

$$
T(u)=\sum_{\sigma \in S_{n}} \operatorname{sgn} \sigma(u+E)_{\sigma(1), 1} \cdots(u+E-n+1)_{\sigma(n), n} .
$$

It is well known that all its coefficients belong to the center of $\mathrm{U}\left(\mathfrak{g}_{n}\right)$ (and generate the center); see e.g. [9]. This also easily follows from the properties the quantum determinant of the Yangian for the Lie algebra $\mathfrak{g l}(n)$; see e.g. [16]. Therefore, these coefficients act in $L(\lambda)$ as scalars which can be easily found by applying $T(u)$ to the highest vector $\xi$ :

$$
\left.T(u)\right|_{L(\lambda)}=\left(u+l_{1}\right) \cdots\left(u+l_{n}\right)
$$

On the other hand, the center of $\mathrm{U}\left(\mathfrak{g}_{n}\right)$ is a subalgebra in the normalizer Norm J. We shall keep the same notation for the image of $T(u)$ in the transvector algebra $\mathrm{Z}\left(\mathfrak{g}_{n}, \mathfrak{g}_{n-1}\right)$. To get explicit expressions of the coefficients of $T(u)$ in terms of the raising and lowering operators we consider $T(u)$ modulo the ideal $\mathrm{J}$ and apply the projection $p$. The details can be found in [15, Theorem 3.1]. We have

$$
T(u)=\left(u+E_{n n}\right) \prod_{i=1}^{n-1}\left(u+h_{i}-1\right)-\sum_{i=1}^{n-1} z_{i n} z_{n i} \prod_{j=1, j \neq i}^{n-1} \frac{u+h_{j}-1}{h_{i}-h_{j}} .
$$

In particular, $T\left(-h_{i}+1\right)=(-1)^{n-1} z_{i n} z_{n i}$.

Now, (2.10) implies that $z_{i n} \xi_{\mu}=0$ unless $\lambda_{i}-\mu_{i} \geq 1$. In this case using (2.9) we obtain

$$
z_{i n} \xi_{\mu}=z_{i n} z_{n i} \xi_{\mu+\delta_{i}}=(-1)^{n-1} T\left(-h_{i}+1\right) \xi_{\mu+\delta_{i}}=(-1)^{n-1} T\left(-m_{i}\right) \xi_{\mu+\delta_{i}},
$$


where we have used $h_{i} \xi_{\mu}=m_{i} \xi_{\mu}$. The relation (2.12) now follows from (2.13).

Applying appropriate raising operators to the vector $\xi_{\mu}$ we can obtain the highest vector $\xi$ of $L(\lambda)$ with a nonzero coefficient. This proves that $\xi_{\mu} \neq 0$.

Given a Gelfand-Tsetlin pattern $\Lambda$ introduce the vector $\xi_{\Lambda} \in L(\lambda)$ by

$$
\xi_{\Lambda}=\prod_{k=2, \ldots, n}\left(z_{k 1}^{\lambda_{k 1}-\lambda_{k-1,1}} \cdots z_{k, k-1}^{\lambda_{k, k-1}-\lambda_{k-1, k-1}}\right) \xi
$$

Lemma 2.2 implies

Corollary 2.3 The vectors $\xi_{\Lambda}$ parametrized by the patterns $\Lambda$ form a basis of the representation $L(\lambda)$.

We now briefly outline a derivation of formulas (2.3)-(2.5) which is standard; see e.g. 25]. First, since $E_{n n} z_{n i}=z_{n i}\left(E_{n n}+1\right)$ for any $i$, we have

$$
E_{n n} \xi_{\mu}=\left(\sum_{i=1}^{n} \lambda_{i}-\sum_{i=1}^{n-1} \mu_{i}\right) \xi_{\mu}
$$

which implies (2.3). To prove 2.4) is suffices to find $E_{n-1, n} \xi_{\mu \nu}$ where

$$
\xi_{\mu \nu}=z_{n-1,1}^{\mu_{1}-\nu_{1}} \cdots z_{n-1, n-2}^{\mu_{n-2}-\nu_{n-2}} \xi_{\mu}
$$

and the $\nu_{i}$ satisfy

$$
\mu_{i}-\nu_{i} \in \mathbb{Z}_{+} \quad \text { and } \quad \nu_{i}-\mu_{i+1} \in \mathbb{Z}_{+} \quad \text { for } \quad i=1, \ldots, n-2 .
$$

Since $E_{n-1, n}$ commutes with the $z_{n-1, i}$,

$$
E_{n-1, n} \xi_{\mu \nu}=z_{n-1,1}^{\mu_{1}-\nu_{1}} \cdots z_{n-1, n-2}^{\mu_{n-2}-\nu_{n-2}} E_{n-1, n} \xi_{\mu} .
$$

Now use the following identity in $\mathrm{U}^{\prime}\left(\mathfrak{g}_{n}\right)$ modulo the ideal $\mathrm{J}$ 25]

$$
E_{n-1, n}=\sum_{i=1}^{n-1} z_{n-1, i} z_{i n} \frac{1}{\left(h_{i}-h_{1}\right) \cdots \wedge \cdots\left(h_{i}-h_{n-1}\right)},
$$

where $z_{n-1, n-1}:=1$. Applying (2.12) we find that

$$
E_{n-1, n} \xi_{\mu \nu}=-\sum_{i=1}^{n-1} \frac{\left(m_{i}-l_{1}\right) \cdots\left(m_{i}-l_{n}\right)}{\left(m_{i}-m_{1}\right) \cdots \wedge \cdots\left(m_{i}-m_{n-1}\right)} \xi_{\mu+\delta_{i}, \nu}
$$


which proves (2.4). To prove (2.5) we use a contravariant bilinear form $\langle$,$\rangle on L(\lambda)$ uniquely determined by the conditions:

$$
\langle\xi, \xi\rangle=1, \quad\left\langle E_{i j} \eta, \zeta\right\rangle=\left\langle\eta, E_{j i} \zeta\right\rangle, \quad \eta, \zeta \in L(\lambda)
$$

The basis $\left\{\xi_{\Lambda}\right\}$ is orthogonal with respect to this form. This follows from (2.12) and the fact that the operators $p E_{i n}$ and $p E_{n i}$ are adjoint to each other with respect to the restriction of the form $\langle$,$\rangle to the space L(\lambda)^{+}$. In particular, (2.15) implies that

$$
E_{n, n-1} \xi_{\mu \nu}=\sum_{i=1}^{n-1} c_{i}(\mu, \nu) \xi_{\mu-\delta_{i}, \nu}
$$

for some coefficients $c_{i}(\mu, \nu)$. Apply the operator $z_{j, n-1}$ to both sides of this relation. Since $z_{j, n-1}$ commutes with $E_{n, n-1}$ we obtain from (2.12) a recurrence relation for the $c_{i}(\mu, \nu):$ if $\mu_{j}-\nu_{j} \geq 1$ then

$$
c_{i}\left(\mu, \nu+\delta_{j}\right)=c_{i}(\mu, \nu) \frac{m_{i}-\gamma_{j}-1}{m_{i}-\gamma_{j}},
$$

where $\gamma_{j}=\nu_{j}-j+1$. This proves (2.5) by induction.

Note that the original Gelfand-Tsetlin basis [5] is orthonormal. The basis vectors in [5] coincide with the $\xi_{\Lambda}$ up to a norm factor which can be explicitly calculated; see e.g. [25].

\section{A basis for odd orthogonal Lie algebras}

We shall enumerate the rows and columns of $(2 n+1) \times(2 n+1)$-matrices over $\mathbb{C}$ by the indices $-n, \ldots,-1,0,1, \ldots, n$.

\subsection{Main theorem}

We keep the notation $E_{i j}, i, j=-n, \ldots, n$ for the standard basis of the Lie algebra $\mathfrak{g l}(2 n+1)$. Introduce the elements

$$
F_{i j}=E_{i j}-E_{-j,-i}
$$

We have $F_{-j,-i}=-F_{i j}$. In particular, $F_{-i, i}=0$ for all $i$. The orthogonal Lie algebra $\mathfrak{g}_{n}:=\mathfrak{o}(2 n+1)$ can be identified with the subalgebra in $\mathfrak{g l}(2 n+1)$ spanned by the 
elements $F_{i j}, i, j=-n, \ldots, n$. The subalgebra $\mathfrak{g}_{n-1}$ is spanned by the elements (3.1) with the indices $i, j$ running over the set $\{-n+1, \ldots, n-1\}$. Denote by $\mathfrak{h}=\mathfrak{h}_{n}$ the diagonal Cartan subalgebra in $\mathfrak{g}_{n}$. The elements $F_{11}, \ldots, F_{n n}$ form a basis of $\mathfrak{h}$.

The finite-dimensional irreducible representations of $\mathfrak{g}_{n}$ are in a one-to-one correspondence with $n$-tuples $\lambda=\left(\lambda_{1}, \ldots, \lambda_{n}\right)$ where all the entries $\lambda_{i}$ are simultaneously integers or half-integers (elements of the set $\frac{1}{2}+\mathbb{Z}$ ) and the following inequalities hold:

$$
0 \geq \lambda_{1} \geq \lambda_{2} \geq \cdots \geq \lambda_{n}
$$

Such an $n$-tuple $\lambda$ is called the highest weight of the corresponding representation which we shall denote by $V(\lambda)$. It contains a unique, up to a multiple, nonzero vector $\xi$ (the highest vector) such that $F_{i i} \xi=\lambda_{i} \xi$ for $i=1, \ldots, n$ and $F_{i j} \xi=0$ for $-n \leq i<j \leq n$. Denote by $V(\lambda)^{+}$the subspace of $\mathfrak{g}_{n-1}$-highest vectors in $V(\lambda)$ :

$$
V(\lambda)^{+}=\left\{\eta \in V(\lambda) \mid F_{i j} \eta=0, \quad-n<i<j<n\right\} .
$$

Given a $\mathfrak{g}_{n-1}$-highest weight $\mu=\left(\mu_{1}, \ldots, \mu_{n-1}\right)$ we denote by $V(\lambda)_{\mu}^{+}$the corresponding weight subspace in $V(\lambda)^{+}$:

$$
V(\lambda)_{\mu}^{+}=\left\{\eta \in V(\lambda)^{+} \mid F_{i i} \eta=\mu_{i} \eta, \quad i=1, \ldots, n-1\right\} .
$$

By the branching rule for the reduction $\mathfrak{g}_{n} \downarrow \mathfrak{g}_{n-1}$ [24] we have

$$
\left.V(\lambda)\right|_{\mathfrak{g}_{n-1}} \simeq \bigoplus c(\mu) V^{\prime}(\mu)
$$

where $V^{\prime}(\mu)$ is the irreducible finite-dimensional representation of $\mathfrak{g}_{n-1}$ with the highest weight $\mu$, and $c(\mu)$ equals the number of $n$-tuples $\rho=\left(\rho_{1}, \ldots, \rho_{n}\right)$ satisfying the inequalities

$$
\begin{aligned}
& -\lambda_{1} \geq \rho_{1} \geq \lambda_{1} \geq \rho_{2} \geq \lambda_{2} \geq \cdots \geq \rho_{n-1} \geq \lambda_{n-1} \geq \rho_{n} \geq \lambda_{n}, \\
& -\mu_{1} \geq \rho_{1} \geq \mu_{1} \geq \rho_{2} \geq \mu_{2} \geq \cdots \geq \rho_{n-1} \geq \mu_{n-1} \geq \rho_{n}
\end{aligned}
$$

with all the $\rho_{i}$ and $\mu_{i}$ being simultaneously integers or half-integers together with the $\lambda_{i}$. Any nonzero vector in $V(\lambda)_{\mu}^{+}$generates a $\mathfrak{g}_{n-1}$-submodule in $V(\lambda)$ isomorphic to $V^{\prime}(\mu)$. We obviously have $\operatorname{dim} V(\lambda)_{\mu}^{+}=c(\mu)$. Basis vectors in $V(\lambda)_{\mu}^{+}$can be parametrized by the $n$-tuples $\rho$. We shall be using an equivalent parameterization by $(n+1)$-tuples $\nu=\left(\sigma, \nu_{1}, \ldots, \nu_{n}\right)$, where $\nu_{i}=\rho_{i}$ for $i \geq 2$, and

$$
\left(\sigma, \nu_{1}\right)= \begin{cases}\left(0, \rho_{1}\right) & \text { if } \rho_{1} \leq 0 \\ \left(1,-\rho_{1}\right) & \text { if } \rho_{1}>0\end{cases}
$$


A parameterization of basis vectors in $V(\lambda)$ is obtained by using its subsequent restrictions to the subalgebras of the chain $\mathfrak{g}_{1} \subset \mathfrak{g}_{2} \subset \cdots \subset \mathfrak{g}_{n-1} \subset \mathfrak{g}_{n}$. Define a pattern $\Lambda$ associated with $\lambda$ as an array of the form

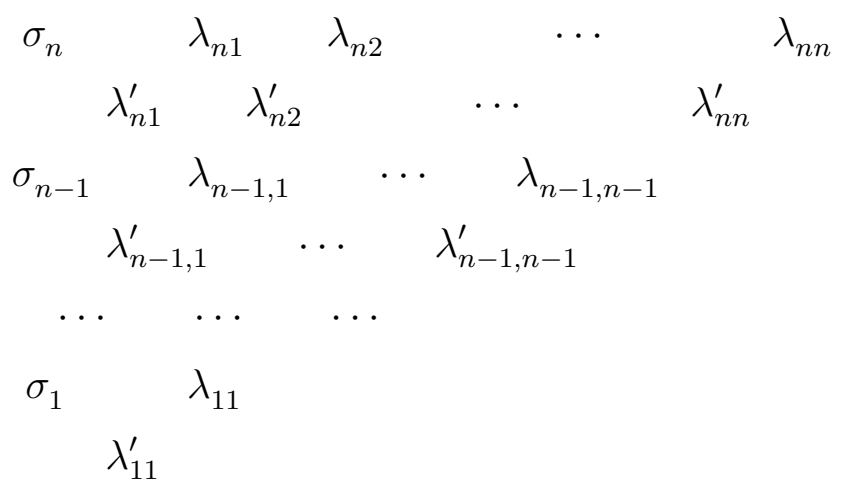

such that $\lambda=\left(\lambda_{n 1}, \ldots, \lambda_{n n}\right)$, each $\sigma_{k}$ is 0 or 1 , the remaining entries are all nonpositive integers or non-positive half-integers together with the $\lambda_{i}$, and the following inequalities hold

$$
\lambda_{k 1}^{\prime} \geq \lambda_{k 1} \geq \lambda_{k 2}^{\prime} \geq \lambda_{k 2} \geq \cdots \geq \lambda_{k, k-1}^{\prime} \geq \lambda_{k, k-1} \geq \lambda_{k k}^{\prime} \geq \lambda_{k k}
$$

for $k=1, \ldots, n$; and

$$
\lambda_{k 1}^{\prime} \geq \lambda_{k-1,1} \geq \lambda_{k 2}^{\prime} \geq \lambda_{k-1,2} \geq \cdots \geq \lambda_{k, k-1}^{\prime} \geq \lambda_{k-1, k-1} \geq \lambda_{k k}^{\prime}
$$

for $k=2, \ldots, n$; in addition, in the case of integer $\lambda_{i}$ the condition

$$
\lambda_{k 1}^{\prime} \leq-1 \quad \text { if } \quad \sigma_{k}=1
$$

should hold for all $k=1, \ldots, n$. Let us set $l_{k 0}=-1 / 2$ for all $k$, and

$$
l_{k i}=\lambda_{k i}-i+1 / 2, \quad l_{k i}^{\prime}=\lambda_{k i}^{\prime}-i+1 / 2, \quad 1 \leq i \leq k \leq n .
$$

Given a pattern $\Lambda$ set for $i=0,1, \ldots, k-1$

$$
A_{k i}=\prod_{a=1, a \neq i}^{k-1} \frac{1}{l_{k-1, i}-l_{k-1, a}} \cdot \prod_{a=1}^{k-1} \frac{1}{l_{k-1, i}+l_{k-1, a}} .
$$

Furthermore, introduce polynomials $B_{k i}(x)$ by

$$
B_{k i}(x)=\prod_{a=1, a \neq i}^{k} \frac{\left(x+l_{k a}^{\prime}+1\right)\left(x-l_{k a}^{\prime}\right)}{l_{k a}^{\prime}-l_{k i}^{\prime}},
$$


and define the numbers $C_{k i}$ by

$$
C_{k i}=l_{k i}^{\prime}\left(1-2 \sigma_{k}-2 l_{k i}^{\prime}\right) \prod_{a=1}^{k}\left(l_{k a}-l_{k i}^{\prime}\right) \prod_{a=1}^{k-1}\left(l_{k-1, a}-l_{k i}^{\prime}\right) \prod_{a=1, a \neq i}^{k} \frac{1}{l_{k a}^{\prime}-l_{k i}^{\prime}} .
$$

We denote by $\Lambda \pm \delta_{k i}$ and $\Lambda+\delta_{k i}^{\prime}$ the arrays obtained from $\Lambda$ by replacing $\lambda_{k i}$ and $\lambda_{k i}^{\prime}$ by $\lambda_{k i} \pm 1$ and $\lambda_{k i}^{\prime}+1$ respectively.

The following is our main theorem which will be proved in Sections 3.4 and 3.5.

Theorem 3.1 There exists a basis $\left\{\zeta_{\Lambda}\right\}$ of $V(\lambda)$ parametrized by the patterns $\Lambda$ such that the action of the generators of $\mathfrak{g}_{n}$ is given by the formulas

$$
\begin{aligned}
F_{k k} \zeta_{\Lambda} & =\left(\sigma_{k}+2 \sum_{i=1}^{k} \lambda_{k i}^{\prime}-\sum_{i=1}^{k} \lambda_{k i}-\sum_{i=1}^{k-1} \lambda_{k-1, i}\right) \zeta_{\Lambda}, \\
F_{k-1,-k} \zeta_{\Lambda} & =A_{k 0} \zeta_{\Lambda}(k, 0)+\sum_{i=1}^{k-1} A_{k i}\left(\frac{1}{l_{k-1, i}+1 / 2} \zeta_{\Lambda}^{+}(k, i)-\frac{1}{l_{k-1, i}-1 / 2} \zeta_{\Lambda}^{-}(k, i)\right) .
\end{aligned}
$$

Here the following notation has been used

$$
\begin{aligned}
& \zeta_{\Lambda}^{-}(k, i)=\zeta_{\Lambda-\delta_{k-1, i}} \\
& \zeta_{\Lambda}^{+}(k, i)=\sum_{j=1}^{k} \sum_{m=1}^{k-1} B_{k j}\left(l_{k-1, i}\right) B_{k-1, m}\left(l_{k-1, i}\right) \zeta_{\Lambda+\delta_{k j}^{\prime}+\delta_{k-1, i}+\delta_{k-1, m}^{\prime}}
\end{aligned}
$$

and $\zeta_{\Lambda}:=0$ if $\Lambda$ is not a pattern. Furthermore,

$$
\begin{aligned}
& \zeta_{\Lambda}(k, 0)=(-1)^{k} \zeta_{\bar{\Lambda}} \quad \text { if } \sigma_{k}=\sigma_{k-1}=0, \\
& =\sum_{j=1}^{k} B_{k j}\left(l_{k-1,0}\right) \zeta_{\bar{\Lambda}+\delta_{k j}^{\prime}} \quad \text { if } \sigma_{k}=1, \sigma_{k-1}=0 \text {, } \\
& =-\sum_{m=1}^{k-1} B_{k-1, m}\left(l_{k-1,0}\right) \zeta_{\bar{\Lambda}+\delta_{k-1, m}^{\prime}} \quad \text { if } \sigma_{k}=0, \sigma_{k-1}=1 \text {, } \\
& =(-1)^{k-1} \sum_{j=1}^{k} \sum_{m=1}^{k-1} B_{k j}\left(l_{k-1,0}\right) B_{k-1, m}\left(l_{k-1,0}\right) \zeta_{\bar{\Lambda}+\delta_{k j}^{\prime}+\delta_{k-1, m}^{\prime}} \\
& \text { if } \sigma_{k}=\sigma_{k-1}=1 \text {, }
\end{aligned}
$$

where $\bar{\Lambda}$ is obtained from $\Lambda$ by replacing $\sigma_{k}$ and $\sigma_{k-1}$ respectively with $\sigma_{k}+1$ and $\sigma_{k-1}+1$ (modulo 2). The action of $F_{k-1, k}$ is found from the relation

$$
F_{k-1, k}=\left[\Phi_{k-1,-k}(u+2) \Phi_{-k, k}-\Phi_{-k, k} \Phi_{k-1,-k}(u)\right]_{u=0}
$$


where the operator $\Phi_{-k, k}$ acts on the basis elements by the rule

$$
\Phi_{-k, k} \zeta_{\Lambda}=\sum_{i=1}^{k} C_{k i}\left(F_{k k}-l_{k i}^{\prime}+1\right) \zeta_{\Lambda-\delta_{k i}^{\prime}}
$$

while the action of $\Phi_{k-1,-k}(u)$ is given by

$$
\begin{aligned}
\Phi_{k-1,-k}(u) \zeta_{\Lambda} & =\frac{A_{k 0}}{u+F_{k k}-3 / 2} \zeta_{\Lambda}(k, 0) \\
+ & \sum_{i=1}^{k-1} A_{k i}\left(\frac{1}{\left(l_{k-1, i}+1 / 2\right)\left(u+l_{k-1, i}+F_{k k}-1\right)} \zeta_{\Lambda}^{+}(k, i)\right. \\
& \left.\quad-\frac{1}{\left(l_{k-1, i}-1 / 2\right)\left(u-l_{k-1, i}+F_{k k}-1\right)} \zeta_{\Lambda}^{-}(k, i)\right) .
\end{aligned}
$$

Remark. The image of $\zeta_{\Lambda}$ under the operator $\Phi_{k-1,-k}(u+2) \Phi_{-k, k}-\Phi_{-k, k} \Phi_{k-1,-k}(u)$ at $u=0$ may be undefined for some patterns $\Lambda$. To get the action of $F_{k-1, k}$, one should first calculate its matrix elements in a "generic" representation $V(\lambda)$ and then specialize the parameters; see Section 3.5. For example, consider the case $n=1$. It will be shown in Section 3.4 that the basis vectors in $V(\lambda)$ are given by

$$
\zeta_{\Lambda}=F_{10}^{\sigma_{1}}\left(F_{10} F_{0,-1}\right)^{\lambda_{11}^{\prime}-\lambda_{11}} \xi
$$

Furthermore, the operators $\Phi_{-1,1}$ and $\Phi_{0,-1}(u)$ are defined by

$$
\begin{aligned}
\Phi_{-1,1} & =-\frac{1}{2} F_{01}^{2} \\
\Phi_{0,-1}(u) & =F_{0,-1} \frac{1}{u+F_{11}-1 / 2}
\end{aligned}
$$

see Section 3.5. In the case $\lambda=(-1 / 2)$ the basis of $V(\lambda)$ consists of two vectors $\xi$ and $\xi^{\prime}=F_{10} \xi$. Therefore, $\Phi_{-1,1}$ is the zero operator in $V(\lambda)$ while the image $\Phi_{0,-1}(0) \xi^{\prime}$ is not defined. On the other hand, we find directly that $F_{01} \xi^{\prime}=1 / 2 \xi$.

\subsection{Transvector algebra $\mathrm{Z}\left(\mathfrak{g}_{n}, \mathfrak{g}_{n-1}\right)$}

Consider the extension of the universal enveloping algebra $\mathrm{U}\left(\mathfrak{g}_{n}\right)$

$$
\mathrm{U}^{\prime}\left(\mathfrak{g}_{n}\right)=\mathrm{U}\left(\mathfrak{g}_{n}\right) \otimes_{\mathrm{U}(\mathfrak{h})} \mathrm{R}(\mathfrak{h})
$$


where $R(\mathfrak{h})$ is the field of fractions of the commutative algebra $U(\mathfrak{h})$. Let $J$ denote the left ideal in $\mathrm{U}^{\prime}\left(\mathfrak{g}_{n}\right)$ generated by the elements $F_{i j}$ with $-n<i<j<n$. The transvector algebra $\mathrm{Z}\left(\mathfrak{g}_{n}, \mathfrak{g}_{n-1}\right)$ is the quotient algebra of the normalizer

$$
\text { Norm } \mathrm{J}=\left\{x \in \mathrm{U}^{\prime}\left(\mathfrak{g}_{n}\right) \mid \mathrm{J} x \subseteq \mathrm{J}\right\}
$$

modulo the two-sided ideal $\mathrm{J}$ [26]. It is an algebra over $\mathbb{C}$ and an $\mathrm{R}(\mathfrak{h})$-bimodule. Let $p=p_{n-1}$ denote the extremal projection for the Lie algebra $\mathfrak{g}_{n-1}$ [1, 26]; cf. Section 2 . It satisfies the following (characteristic) relations

$$
F_{i j} p=p F_{j i}=0 \quad \text { for } \quad-n<i<j<n \text {. }
$$

The projection $p$ naturally acts in the space $\mathrm{U}^{\prime}\left(\mathfrak{g}_{n}\right) / \mathrm{J}$ and its image coincides with $\mathrm{Z}\left(\mathfrak{g}_{n}, \mathfrak{g}_{n-1}\right)$. The elements

$$
p F_{i a}=-p F_{-a,-i}, \quad a=-n, n, \quad i=-n+1, \ldots, n-1
$$

are generators of $\mathrm{Z}\left(\mathfrak{g}_{n}, \mathfrak{g}_{n-1}\right)$ [26]. Set

$$
f_{0}=-1 / 2, \quad f_{i}=F_{i i}-i+1 / 2, \quad f_{-i}=-f_{i}
$$

for $i=1, \ldots, n$; and set $f_{i}^{\prime}=-f_{-i}$ for all $i$. The elements (3.4) can be given by the following explicit formulas (modulo $\mathrm{J})$ :

$$
p F_{i a}=\sum_{i>i_{1}>\cdots>i_{s}>-n} F_{i i_{1}} F_{i_{1} i_{2}} \cdots F_{i_{s-1} i_{s}} F_{i_{s} a} \frac{1}{\left(f_{i}-f_{i_{1}}\right) \cdots\left(f_{i}-f_{i_{s}}\right)},
$$

where $s=0,1, \ldots$ We shall use normalized generators of $\mathrm{Z}\left(\mathfrak{g}_{n}, \mathfrak{g}_{n-1}\right)$ defined by

$$
\begin{aligned}
& z_{i a}=p F_{i a}\left(f_{i}-f_{i-1}\right) \cdots\left(f_{i}-f_{-n+1}\right) \\
& z_{a i}=p F_{a i}\left(f_{i}^{\prime}-f_{i+1}^{\prime}\right) \cdots\left(f_{i}^{\prime}-f_{n-1}^{\prime}\right)
\end{aligned}
$$

We obviously have $z_{a i}=(-1)^{n-i} z_{-i,-a}$. The elements $z_{i a}$ satisfy certain quadratic relations [26]. We shall use the following ones below (cf. (2.9) and (2.10)): for $a, b \in\{-n, n\}$ and $i+j \neq 0$ one has

$$
z_{a j} z_{b i}\left(f_{i}^{\prime}-f_{j}^{\prime}+1\right)=z_{b i} z_{a j}\left(f_{i}^{\prime}-f_{j}^{\prime}\right)+z_{a i} z_{b j}
$$

In particular, $z_{a i}$ and $z_{a j}$ commute for $i+j \neq 0$. One easily verifies that $z_{a i}$ and $z_{b i}$ also commute for $i \neq 0$ and all $a, b$. 
The elements $z_{i a}$ and $z_{a i}$ naturally act in the space $V(\lambda)^{+}$and are called the raising and lowering operators. One has for $i=1, \ldots, n-1$ :

$$
z_{i a}: V(\lambda)_{\mu}^{+} \rightarrow V(\lambda)_{\mu+\delta_{i}}^{+}, \quad z_{a i}: V(\lambda)_{\mu}^{+} \rightarrow V(\lambda)_{\mu-\delta_{i}}^{+},
$$

where $\mu \pm \delta_{i}$ is obtained from $\mu$ by replacing $\mu_{i}$ with $\mu_{i} \pm 1$. The operators $z_{0 a}$ preserve each subspace $V(\lambda)_{\mu}^{+}$.

We shall need the following element which can be checked to belong to the normalizer Norm J, and so it can be regarded as an element of the algebra $\mathrm{Z}\left(\mathfrak{g}_{n}, \mathfrak{g}_{n-1}\right)$ :

$$
z_{n,-n}=\sum_{n>i_{1}>\cdots>i_{s}>-n} F_{n i_{1}} F_{i_{1} i_{2}} \cdots F_{i_{s},-n}\left(f_{n}-f_{j_{1}}\right) \cdots\left(f_{n}-f_{j_{k}}\right)
$$

where $s=1,2, \ldots$ and $\left\{j_{1}, \ldots, j_{k}\right\}$ is the complement to the subset $\left\{i_{1}, \ldots, i_{s}\right\}$ in $\{-n+1, \ldots, n-1\}$. The following relation is proved exactly as its $C$ and $D$ series counterparts 13, 14 (cf. (2.14)): for $a=-n, n$

$$
F_{n-1, a}=\sum_{i=-n+1}^{n-1} z_{n-1, i} z_{i a} \frac{1}{\left(f_{i}-f_{-n+1}\right) \cdots \wedge \cdots\left(f_{i}-f_{n-1}\right)},
$$

where $z_{n-1, n-1}:=1$ and the equalities are considered in $\mathrm{U}^{\prime}\left(\mathfrak{g}_{n}\right)$ modulo the ideal $\mathrm{J}$.

\subsection{Yangians and twisted Yangians}

Let us introduce the $\mathfrak{g l}(2)$ - Yangian $\mathrm{Y}(2)$ and the (orthogonal) twisted Yangian $\mathrm{Y}^{+}(2)$; see [16] for more details. The Yangian $\mathrm{Y}(2)$ is the complex associative algebra with the generators $t_{a b}^{(1)}, t_{a b}^{(2)}, \ldots$ where $a, b \in\{-n, n\}$, and the defining relations

$$
\left[t_{a b}(u), t_{c d}(v)\right]=\frac{1}{u-v}\left(t_{c b}(u) t_{a d}(v)-t_{c b}(v) t_{a d}(u)\right)
$$

where

$$
t_{a b}(u):=\delta_{a b}+t_{a b}^{(1)} u^{-1}+t_{a b}^{(2)} u^{-2}+\cdots \in \mathrm{Y}(2)\left[\left[u^{-1}\right]\right]
$$

Introduce the series $s_{a b}(u), a, b \in\{-n, n\}$ by

$$
s_{a b}(u)=t_{a n}(u) t_{-b,-n}(-u)+t_{a,-n}(u) t_{-b, n}(-u) .
$$

Write $s_{a b}(u)=\delta_{a b}+s_{a b}^{(1)} u^{-1}+s_{a b}^{(2)} u^{-2}+\cdots$. The twisted Yangian $\mathrm{Y}^{+}(2)$ is defined as the subalgebra of $\mathrm{Y}(2)$ generated by the elements $s_{a b}^{(1)}, s_{a b}^{(2)}, \ldots$ where $a, b \in\{-n, n\}$. Also, 
$\mathrm{Y}^{+}(2)$ can be viewed as an abstract algebra with generators $s_{a b}^{(r)}$ and the following defining relations (see [16, Section 3]):

$$
\begin{aligned}
{\left[s_{a b}(u), s_{c d}(v)\right] } & =\frac{1}{u-v}\left(s_{c b}(u) s_{a d}(v)-s_{c b}(v) s_{a d}(u)\right) \\
& -\frac{1}{u+v}\left(s_{a,-c}(u) s_{-b, d}(v)-s_{c,-a}(v) s_{-d, b}(u)\right) \\
& +\frac{1}{u^{2}-v^{2}}\left(s_{c,-a}(u) s_{-b, d}(v)-s_{c,-a}(v) s_{-b, d}(u)\right)
\end{aligned}
$$

and

$$
s_{-b,-a}(-u)=\frac{2 u+1}{2 u} s_{a b}(u)-\frac{1}{2 u} s_{a b}(-u) .
$$

The Yangian $\mathrm{Y}(2)$ is a Hopf algebra with the coproduct

$$
\Delta\left(t_{a b}(u)\right)=t_{a n}(u) \otimes t_{n b}(u)+t_{a,-n}(u) \otimes t_{-n, b}(u)
$$

The twisted Yangian $\mathrm{Y}^{+}(2)$ is a left coideal in $\mathrm{Y}(2)$ with

$$
\Delta\left(s_{a b}(u)\right)=\sum_{c, d \in\{-n, n\}} t_{a c}(u) t_{-b,-d}(-u) \otimes s_{c d}(u) .
$$

Given a pair of complex numbers $(\alpha, \beta)$ such that $\alpha-\beta \in \mathbb{Z}_{+}$we denote by $L(\alpha, \beta)$ the irreducible representation of the Lie algebra $\mathfrak{g l}(2)$ with the highest weight $(\alpha, \beta)$ with respect to the upper triangular Borel subalgebra; see Section 2. We have $\operatorname{dim} L(\alpha, \beta)=\alpha-\beta+1$. We may regard $L(\alpha, \beta)$ as a $\mathrm{Y}(2)$-module by using the algebra homomorphism $\mathrm{Y}(2) \rightarrow \mathrm{U}(\mathfrak{g l}(2))$ given by

$$
t_{a b}(u) \mapsto \delta_{a b}+E_{a b} u^{-1}, \quad a, b \in\{-n, n\} .
$$

The coproduct (3.14) allows one to construct representations of $\mathrm{Y}(2)$ of the form

$$
L=L\left(\alpha_{1}, \beta_{1}\right) \otimes \cdots \otimes L\left(\alpha_{n}, \beta_{n}\right)
$$

Note that the generators $t_{a b}^{(r)}$ with $r>n$ act as zero operators in $L$. Therefore, the operators $T_{a b}(u)=u^{n} t_{a b}(u)$ are polynomials in $u$ :

$$
T_{a b}(u)=\delta_{a b} u^{n}+t_{a b}^{(1)} u^{n-1}+\cdots+t_{a b}^{(n)} .
$$

For any $\gamma \in \mathbb{C}$ denote by $W(\gamma)$ the one-dimensional representation of $\mathrm{Y}^{+}(2)$ spanned by a vector $w$ such that

$$
s_{n n}(u) w=\frac{u+\gamma}{u+1 / 2} w, \quad s_{-n,-n}(u) w=\frac{u-\gamma+1}{u+1 / 2} w
$$


and $s_{a,-a}(u) w=0$ for $a=-n, n$. By (3.15) we can regard the tensor product $L \otimes W(\gamma)$ as a representation of $\mathrm{Y}^{+}(2)$. Representations of this type essentially exhaust all finite-dimensional irreducible representations of $\mathrm{Y}^{+}(2)$ [12]. The vector space isomorphism

$$
L \otimes W(\gamma) \rightarrow L, \quad v \otimes w \mapsto v, \quad v \in L
$$

provides $L \otimes W(\gamma)$ with an action of $\mathrm{Y}(2)$.

\subsection{Construction of the basis}

Introduce the following polynomials in $u$ with coefficients in the transvector algebra $\mathrm{Z}\left(\mathfrak{g}_{n}, \mathfrak{g}_{n-1}\right):$ for $a, b \in\{-n, n\}$

$$
Z_{a b}(u)=-\left(\delta_{a b}(u-n+1)+F_{a b}\right) \prod_{i=-n+1}^{n-1}\left(u+g_{i}\right)+\sum_{i=-n+1}^{n-1} z_{a i} z_{i b} \prod_{j=-n+1, j \neq i}^{n-1} \frac{u+g_{j}}{g_{i}-g_{j}}
$$

where $g_{i}:=f_{i}+1 / 2$ for all $i$.

Proposition 3.2 The mapping

$$
s_{a b}(u) \mapsto-u^{-2 n} Z_{a b}(u), \quad a, b \in\{-n, n\}
$$

defines an algebra homomorphism $\mathrm{Y}^{+}(2) \rightarrow \mathrm{Z}\left(\mathfrak{g}_{n}, \mathfrak{g}_{n-1}\right)$.

Proof. One of the possible ways to prove the claim is to check directly that the relations (3.12) and (3.13) are satisfied with the $s_{a b}(u)$ respectively replaced with $Z_{a b}(u)$. Here one needs to use the quadratic relations in the transvector algebra $\mathrm{Z}\left(\mathfrak{g}_{n}, \mathfrak{g}_{n-1}\right)$. In addition to (3.7) the relations which express $z_{i a} z_{b i}$ in terms of the $z_{b j} z_{j a}$ are needed; see [26.

Alternatively, we can follow the approach of [13, Section 5] to construct first a homomorphism from $\mathrm{Y}^{+}(2)$ to the centralizer $\mathrm{C}_{n}$ of $\mathfrak{g}_{n-1}$ in $\mathrm{U}\left(\mathfrak{g}_{n}\right)$ and then calculate the images of the centralizer elements in the algebra $\mathrm{Z}\left(\mathfrak{g}_{n}, \mathfrak{g}_{n-1}\right)$. The calculation is similar to that in the symplectic case [13]; see also [14]. We shall only give a few key formulas here. Introduce the $(2 n+1) \times(2 n+1)$-matrix $F=\left(F_{i j}\right)$ whose $i j$ th entry is the element $F_{i j} \in \mathfrak{g}_{n}$ and set

$$
F(u)=1+\frac{F}{u+1 / 2} .
$$


Denote by $\widehat{F}(u)$ the corresponding Sklyanin comatrix; see [12, Section 2]. The mapping

$$
s_{a b}(u) \mapsto c(u) \widehat{F}(-u+n-1 / 2)_{a b}, \quad a, b \in\{-n, n\},
$$

where $c(u)=\left(1-u^{-2}\right)\left(1-4 u^{-2}\right) \cdots\left(1-(n-1)^{2} u^{-2}\right)$, defines an algebra homomorphism $\mathrm{Y}^{+}(2) \rightarrow \mathrm{C}_{n}$ [12, Proposition 2.1]; cf. [20]. Its composition with the natural homomorphism $\mathrm{C}_{n} \rightarrow \mathrm{Z}\left(\mathfrak{g}_{n}, \mathfrak{g}_{n-1}\right)$ gives (3.18).

As it follows from the branching rule (3.2), the space $V(\lambda)_{\mu}^{+}$is nonzero only if there exists $\nu$ such that the inequalities (3.3) hold. We shall be assuming that this condition is satisfied. Proposition 3.2 allows one to equip $V(\lambda)_{\mu}^{+}$with a structure of a $\mathrm{Y}^{+}(2)$-module defined via the homomorphism (3.18). The next theorem provides an identification of this module.

Theorem 3.3 The $\mathrm{Y}^{+}(2)$-module $V(\lambda)_{\mu}^{+}$is isomorphic to the direct sum of two irreducible submodules, $V(\lambda)_{\mu}^{+} \simeq U \oplus U^{\prime}$, where

$$
\begin{aligned}
U & =L\left(0, \beta_{1}\right) \otimes L\left(\alpha_{2}, \beta_{2}\right) \otimes \cdots \otimes L\left(\alpha_{n}, \beta_{n}\right) \otimes W(1 / 2), \\
U^{\prime} & =L\left(-1, \beta_{1}\right) \otimes L\left(\alpha_{2}, \beta_{2}\right) \otimes \cdots \otimes L\left(\alpha_{n}, \beta_{n}\right) \otimes W(1 / 2),
\end{aligned}
$$

if the $\lambda_{i}$ are integers (it is supposed that $U^{\prime}=\{0\}$ if $\beta_{1}=0$ ); or

$$
\begin{aligned}
U & =L\left(-1 / 2, \beta_{1}\right) \otimes L\left(\alpha_{2}, \beta_{2}\right) \otimes \cdots \otimes L\left(\alpha_{n}, \beta_{n}\right) \otimes W(0), \\
U^{\prime} & =L\left(-1 / 2, \beta_{1}\right) \otimes L\left(\alpha_{2}, \beta_{2}\right) \otimes \cdots \otimes L\left(\alpha_{n}, \beta_{n}\right) \otimes W(1),
\end{aligned}
$$

if the $\lambda_{i}$ are half-integers, and the following notation is used

$$
\begin{aligned}
\alpha_{i} & =\min \left\{\lambda_{i-1}, \mu_{i-1}\right\}-i+1, & i & =2, \ldots, n, \\
\beta_{i} & =\max \left\{\lambda_{i}, \mu_{i}\right\}-i+1, & i & =1, \ldots, n,
\end{aligned}
$$

with $\mu_{n}:=-\infty$. In particular, each of $U$ and $U^{\prime}$ (and hence $\left.V(\lambda)_{\mu}^{+}\right)$is equipped with an action of $\mathrm{Y}(2)$ defined by (3.16).

Proof. Consider the following two vectors in $V(\lambda)_{\mu}^{+}$

$$
\xi_{\mu}=\prod_{i=1}^{n-1}\left(z_{n i}^{\max \left\{\lambda_{i}, \mu_{i}\right\}-\mu_{i}} z_{i,-n}^{\max \left\{\lambda_{i}, \mu_{i}\right\}-\lambda_{i}}\right) \xi, \quad \xi_{\mu}^{\prime}=z_{n 0} \xi_{\mu} .
$$


Repeating the arguments of the proof of Theorem 5.2 in [13] we can show that both $\xi_{\mu}$ and $\xi_{\mu}^{\prime}$ are eigenvectors for $s_{n n}(u)$ and are annihilated by $s_{-n, n}(u)$. Namely,

$$
s_{n n}(u) \xi_{\mu}=\mu(u) \xi_{\mu}, \quad s_{n n}(u) \xi_{\mu}^{\prime}=\left(1+u^{-1}\right) \mu(u) \xi_{\mu}^{\prime}
$$

where

$$
\mu(u)=\left(1-\alpha_{2} u^{-1}\right) \cdots\left(1-\alpha_{n} u^{-1}\right)\left(1+\beta_{1} u^{-1}\right) \cdots\left(1+\beta_{n} u^{-1}\right) .
$$

This is proved simultaneously with the following relations by induction on the degree of the monomial in (3.24): for $i=1, \ldots, n-1$

$$
z_{i n} \xi_{\mu}=-\left(m_{i}+\alpha_{1}\right) \cdots\left(m_{i}+\alpha_{n}\right)\left(m_{i}-\beta_{1}\right) \cdots\left(m_{i}-\beta_{n}\right) \xi_{\mu+\delta_{i}},
$$

and

$$
z_{-n i} \xi_{\mu}=-\left(m_{i}-\alpha_{1}-1\right) \cdots\left(m_{i}-\alpha_{n}-1\right)\left(m_{i}+\beta_{1}-1\right) \cdots\left(m_{i}+\beta_{n}-1\right) \xi_{\mu-\delta_{i}},
$$

where $\alpha_{1}=0$ and $m_{i}=\mu_{i}-i+1$ for $i=1, \ldots, n-1$. Indeed, we note that if $\mu_{i} \geq \lambda_{i}$ then $z_{\text {in }} \xi_{\mu}=0$ which is implied by (3.7). This agrees with (3.26) because in this case $\beta_{i}=m_{i}$. Now assume that $\mu_{i}<\lambda_{i}$. We have $z_{i n} \xi_{\mu}=z_{i n} z_{n i} \xi_{\mu+\delta_{i}}$ by (3.7). Formula (3.17) gives $z_{i n} z_{n i}=z_{-n,-i} z_{-i,-n}=Z_{-n,-n}\left(-g_{-i}\right)$. Further,

$$
Z_{-n,-n}\left(-g_{-i}\right) \xi_{\mu+\delta_{i}}=Z_{-n,-n}\left(m_{i}\right) \xi_{\mu+\delta_{i}}
$$

By Proposition 3.2 and the symmetry relation (3.13) we can write

$$
Z_{-n,-n}\left(m_{i}\right)=\frac{2 m_{i}-1}{2 m_{i}} Z_{n n}\left(-m_{i}\right)+\frac{1}{2 m_{i}} Z_{n n}\left(m_{i}\right)
$$

By induction, $Z_{n n}(u) \xi_{\mu+\delta_{i}}$ can be found from (3.18) and (3.25) which gives (3.26). The proof of (3.27) is very similar. To prove (3.25) we apply the induction hypotheses to (3.26) and (3.27) and also use the relation

$$
z_{0 n} \xi_{\mu}=0
$$

which is a consequence of (3.7). The relations

$$
Z_{-n, n}(u) \xi_{\mu}=0, \quad Z_{-n, n}(u) \xi_{\mu}^{\prime}=0
$$

follow from (3.17), 3.26) and (3.27). 
Both vectors $\xi_{\mu}$ and $\xi_{\mu}^{\prime}$ are nonzero, except for the case $\beta_{1}=0$ where $\xi_{\mu}^{\prime}=0$. Indeed, applying appropriate operators $z_{i n}$ to $\xi_{\mu}$ or $\xi_{\mu}^{\prime}$ repeatedly, we can obtain the highest vector $\xi$ of $V(\lambda)$ with a nonzero coefficient. It follows from [12, Corollary 6.6] that the tensor products $(3.20)-(3.23)$ are irreducible representations of $\mathrm{Y}^{+}(2)$. An easy calculation shows that the highest weights of the $\mathrm{Y}^{+}(2)$-modules $U$ and $U^{\prime}$ respectively coincide with the $\mathrm{Y}^{+}(2)$-weights of the vectors $\xi_{\mu}$ and $\xi_{\mu}^{\prime}$. So, $U$ and $U^{\prime}$ are respectively isomorphic to quotients of the $\mathrm{Y}^{+}(2)$-submodules in $V(\lambda)_{\mu}^{+}$generated by $\xi_{\mu}$ and $\xi_{\mu}^{\prime}$. On the other hand, the branching rule (3.2) implies that

$$
\operatorname{dim} V(\lambda)_{\mu}^{+}=\operatorname{dim} U+\operatorname{dim} U^{\prime}
$$

Therefore, to complete the proof of the theorem we need to show that the $\mathrm{Y}^{+}(2)$ submodules generated by $\xi_{\mu}$ and $\xi_{\mu}^{\prime}$ are disjoint. For this we employ a contravariant bilinear form $\langle$,$\rangle on V(\lambda)$ uniquely determined by the conditions:

$$
\langle\xi, \xi\rangle=1, \quad\left\langle F_{i j} \eta, \zeta\right\rangle=\left\langle\eta, F_{j i} \zeta\right\rangle, \quad \eta, \zeta \in V(\lambda),
$$

cf. (2.16). One easily shows that its restriction to the subspace $V(\lambda)_{\mu}^{+}$is nondegenerate. Therefore, our claim will follow from the fact that the submodules generated by $\xi_{\mu}$ and $\xi_{\mu}^{\prime}$ are orthogonal to each other with respect to $\langle$,$\rangle . Given an$ operator $A$ in $V(\lambda)^{+}$we denote by $A^{*}$ its adjoint operator with respect to the form:

$$
\langle A \eta, \zeta\rangle=\left\langle\eta, A^{*} \zeta\right\rangle
$$

Since the extremal projection $p$ is stable with respect to the anti-involution $F_{i j} \mapsto F_{j i}$ [26] we derive that $\left(p F_{i a}\right)^{*}=p F_{a i}$ for $a=-n, n$ and $i=-n+1, \ldots, n-1$. Therefore, $z_{i a}^{*}=z_{a i} \cdot c$ where $c$ is an element of $\mathrm{R}\left(\mathfrak{h}_{n-1}\right)$ which can be found from (3.5) and (3.6). This also implies that $\left(z_{a i} z_{i b}\right)^{*}=z_{b i} z_{i a}$ and hence

$$
Z_{a b}(u)^{*}=Z_{b a}(u)
$$

see (3.17). By Proposition 3.2 and the Poincaré-Birkhoff-Witt theorem for the twisted Yangians [16, Remark 3.14], every element of the $\mathrm{Y}^{+}(2)$-submodules generated by $\xi_{\mu}$ and $\xi_{\mu}^{\prime}$ can be written as a linear combination of vectors of the following form, respectively:

$$
Z_{n,-n}\left(u_{1}\right) \cdots Z_{n,-n}\left(u_{k}\right) \xi_{\mu} \quad \text { or } \quad Z_{n,-n}\left(v_{1}\right) \cdots Z_{n,-n}\left(v_{l}\right) \xi_{\mu}^{\prime}
$$

where the $u_{i}$ and $v_{i}$ are complex parameters. Therefore, by (3.29) and (3.30) to prove that the submodules are orthogonal it now suffices to show that $\left\langle\xi_{\mu}, \xi_{\mu}^{\prime}\right\rangle=0$. But 
this follows from (3.28).

Remark. Using Weyl's formula for the dimension of $V(\lambda)$ one can slightly modify the proof of Theorem 3.3 so that the branching rule (3.2) would not be used but follow from the theorem; cf. [13, 14].

It follows from (3.13) that the series $s_{n,-n}(u)$ is even in $u$, and so is the polynomial $Z_{n,-n}(u)$; see Proposition 3.2. On the other hand, (3.17) implies that $Z_{n,-n}\left(-g_{i}\right)=$ $z_{n i} z_{i,-n}$ for $i=1, \ldots, n-1$. Moreover, $Z_{n,-n}\left(-g_{n}\right)=z_{n,-n}$ which follows from (3.8). Since $Z_{n,-n}(u)$ is a polynomial in $u^{2}$ of degree $n-1$, by the Lagrange interpolation formula $Z_{n,-n}(u)$ can also be given by

$$
Z_{n,-n}(u)=\sum_{i=1}^{n} z_{n i} z_{i,-n} \prod_{j=1, j \neq i}^{n} \frac{u^{2}-g_{j}^{2}}{g_{i}^{2}-g_{j}^{2}} .
$$

Remark. To make the above evaluation $Z_{n,-n}\left(-g_{i}\right)$ well-defined we agree to consider the series $Z_{a b}(u)$ with $a, b \in\{-n, n\}$ as elements of the right module over the field of rational functions in $g_{1}, \ldots, g_{n}, u$ generated by monomials in the $z_{i a}$.

Given $\nu$ such that the conditions (3.3) are satisfied, set

$$
\gamma_{i}=\nu_{i}-i+1, \quad l_{i}=\lambda_{i}-i+1, \quad i \geq 1
$$

and introduce the vectors

$$
\xi_{\nu \mu}= \begin{cases}\prod_{i=1}^{n} Z_{n,-n}\left(\gamma_{i}-1\right) \cdots Z_{n,-n}\left(\beta_{i}+1\right) Z_{n,-n}\left(\beta_{i}\right) \xi_{\mu} & \text { if } \sigma=0 \\ \prod_{i=1}^{n} Z_{n,-n}\left(\gamma_{i}-1\right) \cdots Z_{n,-n}\left(\beta_{i}+1\right) Z_{n,-n}\left(\beta_{i}\right) \xi_{\mu}^{\prime} & \text { if } \sigma=1\end{cases}
$$

Using (3.31) and (3.7) we get an equivalent expression; cf. [13, Section 6]:

$$
\xi_{\nu \mu}=z_{n 0}^{\sigma} \prod_{i=1}^{n-1} z_{n i}^{\nu_{i}-\mu_{i}} z_{i,-n}^{\nu_{i}-\lambda_{i}} \cdot \prod_{k=l_{n}}^{\gamma_{n}-1} Z_{n,-n}(k) \xi
$$

Proposition 3.4 The vectors $\xi_{\nu \mu}$ with $\nu$ satisfying (3.3) form a basis of $V(\lambda)_{\mu}^{+}$. 
Proof. Due to Theorem 3.3 it suffices to show that the vectors $\xi_{\nu \mu}$ with $\sigma=0$ form a basis of the subspace $U$ of $V(\lambda)_{\mu}^{+}$while those with $\sigma=1$ form a basis in $U^{\prime}$. Let us write each of the tensor products in (3.20)-(3.23) in the form

$$
L\left(\alpha_{1}, \beta_{1}\right) \otimes L\left(\alpha_{2}, \beta_{2}\right) \otimes \cdots \otimes L\left(\alpha_{n}, \beta_{n}\right) \otimes W\left(-\alpha_{0}\right) .
$$

Regarding this as a Y(2)-module defined by (3.16) we can construct a GelfandTsetlin-type basis in this module as follows. Set

$$
\widetilde{\zeta}_{\nu \mu}=\prod_{i=1}^{n} T_{n,-n}\left(-\gamma_{i}+1\right) \cdots T_{n,-n}\left(-\beta_{i}-1\right) T_{n,-n}\left(-\beta_{i}\right) z_{n 0}^{\sigma} \xi_{\mu} .
$$

The vectors $\widetilde{\zeta}_{\nu \mu}$ with $\nu$ satisfying (3.3) form a basis in the $\mathrm{Y}(2)$-module (3.32); see 22, 11, 19. Furthermore, we have

$$
\begin{aligned}
T_{n n}(u) \widetilde{\zeta}_{\nu \mu} & =\left(u+\gamma_{1}\right) \cdots\left(u+\gamma_{n}\right) \widetilde{\zeta}_{\nu \mu}, \\
T_{n,-n}\left(-\gamma_{i}\right) \widetilde{\zeta}_{\nu \mu} & =\widetilde{\zeta}_{\nu+\delta_{i}, \mu} .
\end{aligned}
$$

We have the following equality of operators in the space (3.32):

$$
Z_{n,-n}(u)=\frac{\left(u-\alpha_{0}\right) T_{n,-n}(-u) T_{n n}(u)+\left(u+\alpha_{0}\right) T_{n,-n}(u) T_{n n}(-u)}{(-1)^{n+1} u}
$$

which is easily derived from (3.10), (3.15) and (3.18). Therefore, by (3.33)

$$
Z_{n,-n}\left(\gamma_{i}\right) \widetilde{\zeta}_{\nu \mu}=-2\left(\alpha_{0}-\gamma_{i}\right) \prod_{a=1, a \neq i}^{n}\left(-\gamma_{a}-\gamma_{i}\right) \widetilde{\zeta}_{\nu+\delta_{i}, \mu}
$$

This shows that for each $\nu$ the vectors $\xi_{\nu \mu}$ and $\widetilde{\zeta}_{\nu \mu}$ coincide up to a nonzero factor.

We shall use the following normalized basis vectors

$$
\zeta_{\nu \mu}=\prod_{1 \leq i<j \leq n}\left(-\gamma_{i}-\gamma_{j}\right) ! \xi_{\nu \mu}
$$

The following formulas for the action of the generators of the Yangian $\mathrm{Y}(2)$ in the basis $\left\{\zeta_{\nu \mu}\right\}$ follow from the above proof: for $i=1, \ldots, n$

$$
\begin{aligned}
T_{n n}(u) \zeta_{\nu \mu} & =\left(u+\gamma_{1}\right) \cdots\left(u+\gamma_{n}\right) \zeta_{\nu \mu}, \\
T_{n,-n}\left(-\gamma_{i}\right) \zeta_{\nu \mu} & =\frac{1}{2\left(\gamma_{i}-\alpha_{0}\right)} \zeta_{\nu+\delta_{i}, \mu}, \\
T_{-n, n}\left(-\gamma_{i}\right) \zeta_{\nu \mu} & =2 \prod_{k=0}^{n}\left(\alpha_{k}-\gamma_{i}+1\right) \prod_{k=1}^{n}\left(\beta_{k}-\gamma_{i}\right) \zeta_{\nu-\delta_{i}, \mu}
\end{aligned}
$$


cf. [13] and [14].

Given a pattern $\Lambda$ (see Section 3.1) introduce the vector

$$
\xi_{\Lambda}=\prod_{k=1, \ldots, n}^{\rightarrow}\left(z_{k 0}^{\sigma_{k}} \cdot \prod_{i=1}^{k-1} z_{k i}^{\lambda_{k i}^{\prime}-\lambda_{k-1, i}} z_{i,-k}^{\lambda_{k i}^{\prime}-\lambda_{k i}} \cdot \prod_{q=l_{k k}}^{l_{k k}^{\prime}-1} Z_{k,-k}\left(q+\frac{1}{2}\right)\right) \xi
$$

and set

$$
\zeta_{\Lambda}=N_{\Lambda} \xi_{\Lambda}, \quad N_{\Lambda}=\prod_{k=2}^{n} \prod_{1 \leq i<j \leq k}\left(-l_{k i}^{\prime}-l_{k j}^{\prime}-1\right) !
$$

The following proposition is implied by the branching rule (3.2) and Proposition 3.4.

Proposition 3.5 The vectors $\zeta_{\Lambda}$ parametrized by the patterns $\Lambda$ form a basis of the representation $V(\lambda)$.

\subsection{Matrix element formulas}

Introduce the following elements of $\mathrm{U}\left(\mathfrak{g}_{n}\right)$ :

$$
\Phi_{-k, k}=\sum_{i=1}^{k-1} F_{-k, i} F_{i k}-\frac{1}{2} F_{0 k}^{2}, \quad k=1, \ldots, n .
$$

We shall find the action of $\Phi_{-k, k}$ in the basis $\left\{\zeta_{\Lambda}\right\}$, which will be used later on. Since $\Phi_{-k, k}$ commutes with the subalgebra $\mathfrak{g}_{k-1}$ it suffices to consider the case $k=$ $n$. The image of $2 \Phi_{-n, n}$ under the natural homomorphism $\pi: \mathrm{C}_{n} \rightarrow \mathrm{Z}\left(\mathfrak{g}_{n}, \mathfrak{g}_{n-1}\right)$ coincides with the coefficient at $u^{2 n-2}$ of the polynomial $Z_{-n, n}(u)$; see the proof of Proposition 3.2. The following equality of operators in (3.32) is obtained from (3.10), (3.15) and (3.18):

$$
Z_{-n, n}(u)=\frac{\left(u-\alpha_{0}\right) T_{-n,-n}(-u) T_{-n, n}(u)+\left(u+\alpha_{0}\right) T_{-n,-n}(u) T_{-n, n}(-u)}{(-1)^{n+1} u} .
$$

Therefore,

$$
\Phi_{-n, n}=-t_{-n, n}^{(2)}+t_{-n, n}^{(1)} t_{-n,-n}^{(1)}+\left(1+\alpha_{0}\right) t_{-n, n}^{(1)} .
$$

The image of $s_{n n}^{(1)}$ under the homomorphism (3.19) is $F_{n n}$. On the other hand, by (3.15) we have

$$
s_{n n}^{(1)}=t_{n n}^{(1)}-t_{-n,-n}^{(1)}-\alpha_{0}-1 / 2,
$$


as operators in the space (3.32). Therefore, (3.35) can be written as

$$
\Phi_{-n, n}=-t_{-n, n}^{(2)}+t_{-n, n}^{(1)} t_{n n}^{(1)}-\left(F_{n n}+3 / 2\right) t_{-n, n}^{(1)} .
$$

Finally, relations (3.34) imply that

$$
\Phi_{-n, n} \zeta_{\nu \mu}=\sum_{i=1}^{n} \theta_{i}\left(F_{n n}-\gamma_{i}+3 / 2\right) \zeta_{\nu-\delta_{i}, \mu}
$$

where

$$
\theta_{i}=-2 \prod_{k=0}^{n}\left(\alpha_{k}-\gamma_{i}+1\right) \prod_{k=1}^{n}\left(\beta_{k}-\gamma_{i}\right) \prod_{j=1, j \neq i}^{n}\left(\gamma_{j}-\gamma_{i}\right)^{-1}
$$

Using the notation in (3.32) we can also write this as

$$
\theta_{i}=\left(2 \gamma_{i}-1\right)\left(1-\sigma-\gamma_{i}\right) \prod_{k=1}^{n}\left(l_{k}-\gamma_{i}\right) \prod_{k=1}^{n-1}\left(m_{k}-\gamma_{i}\right) \prod_{j=1, j \neq i}^{n}\left(\gamma_{j}-\gamma_{i}\right)^{-1}
$$

The action of $F_{n n}$ in $V(\lambda)_{\mu}^{+}$is immediately found so that

$$
F_{n n} \xi_{\nu \mu}=\left(\sigma+2 \sum_{i=1}^{n} \nu_{i}-\sum_{i=1}^{n} \lambda_{i}-\sum_{i=1}^{n-1} \mu_{i}\right) \xi_{\nu \mu}
$$

The operator $F_{n-1,-n}$ preserves the subspace of $\mathfrak{g}_{n-2}$-highest vectors in $V(\lambda)$. Therefore it suffices to calculate its action on the basis vectors of the form

$$
\xi_{\nu \mu \nu^{\prime}}=X_{\mu \nu^{\prime}} \xi_{\nu \mu}
$$

where $X_{\mu \nu^{\prime}}$ denotes the operator

$$
X_{\mu \nu^{\prime}}=z_{n-1,0}^{\sigma^{\prime}} \prod_{i=1}^{n-2} z_{n-1, i}^{\nu_{i}^{\prime}-\mu_{i}^{\prime}} z_{i,-n+1}^{\nu_{i}^{\prime}-\mu_{i}} \cdot \prod_{a=m_{n-1}}^{\gamma_{n-1}^{\prime}-1} Z_{n-1,-n+1}(a)
$$

Here we assume that the conditions (3.3) are satisfied with $\lambda, \nu, \mu$ respectively replaced by $\mu, \nu^{\prime}, \mu^{\prime}$; we have used the notation $\gamma_{i}^{\prime}=\nu_{i}^{\prime}-i+1$. The operator $F_{n-1,-n}$ is permutable with the elements $z_{n-1, i}, z_{i,-n+1}$ and $Z_{n-1,-n+1}(u)$ which follows from their explicit formulas. Hence, we can write

$$
F_{n-1,-n} \xi_{\nu \mu \nu^{\prime}}=X_{\mu \nu^{\prime}} F_{n-1,-n} \xi_{\nu \mu}
$$


Let us apply (3.9) with $a=-n$. We have

$$
f_{i} \xi_{\nu \mu}=\left(m_{i}-1 / 2\right) \xi_{\nu \mu}, \quad f_{-i} \xi_{\nu \mu}=\left(-m_{i}+1 / 2\right) \xi_{\nu \mu}
$$

for $i \geq 1$. Recall also that $f_{0}=-1 / 2$. We now need to express

$$
X_{\mu \nu^{\prime}} z_{n-1, i} z_{i,-n} \xi_{\nu \mu}, \quad i=-n+1, \ldots, n-1
$$

as a linear combination of the vectors $\xi_{\nu \mu \nu^{\prime}}$. Suppose first that $i \geq 1$. Assuming that $\nu_{i}-\mu_{i} \geq 1$ we obtain from (3.7)

$$
z_{i,-n} \xi_{\nu \mu}=z_{i,-n} z_{n i} \xi_{\nu, \mu+\delta_{i}}
$$

By (3.17) this equals

$$
z_{i,-n} z_{n i} \xi_{\nu, \mu+\delta_{i}}=z_{n,-i} z_{-i,-n} \xi_{\nu, \mu+\delta_{i}}=Z_{n,-n}\left(-g_{-i}\right) \xi_{\nu, \mu+\delta_{i}}
$$

We have $-g_{-i} \xi_{\nu, \mu+\delta_{i}}=m_{i} \xi_{\nu, \mu+\delta_{i}}$. Since $Z_{n,-n}\left(\gamma_{p}\right) \xi_{\nu, \mu+\delta_{i}}=\xi_{\nu+\delta_{p}, \mu+\delta_{i}}$ for each $p=$ $1, \ldots, n$, we obtain from the Lagrange interpolation formula that (3.37) takes the form

$$
Z_{n,-n}\left(m_{i}\right) \xi_{\nu, \mu+\delta_{i}}=\sum_{p=1}^{n} \prod_{a=1, a \neq p}^{n} \frac{m_{i}^{2}-\gamma_{a}^{2}}{\gamma_{p}^{2}-\gamma_{a}^{2}} \xi_{\nu+\delta_{p}, \mu+\delta_{i}}
$$

Furthermore, for $i \geq 1$

$$
z_{-i,-n} \xi_{\nu \mu}=(-1)^{n-i} z_{n i} \xi_{\nu \mu}=(-1)^{n-i} \xi_{\nu, \mu-\delta_{i}}
$$

Consider now the vector $z_{0,-n} \xi_{\nu \mu}$. If $\sigma=0$ then it equals

$$
z_{0,-n} \xi_{\nu \mu}=(-1)^{n} z_{n 0} \xi_{\nu \mu}=(-1)^{n} \xi_{\bar{\nu} \mu}
$$

where $\bar{\nu}=\left(\sigma+1, \nu_{1}, \ldots, \nu_{n}\right)$ (addition modulo 2). If $\sigma=1$ then

$$
z_{0,-n} \xi_{\nu \mu}=z_{n 0} z_{0,-n} \xi_{\bar{\nu} \mu}
$$

which coincides with $Z_{n,-n}\left(-g_{0}\right) \xi_{\bar{\nu} \mu}$, where $g_{0}=0$. Using again the Lagrange interpolation formula we find that this equals

$$
Z_{n,-n}\left(m_{0}\right) \xi_{\bar{\nu} \mu}=\sum_{p=1}^{n} \prod_{a=1, a \neq p}^{n} \frac{m_{0}^{2}-\gamma_{a}^{2}}{\gamma_{p}^{2}-\gamma_{a}^{2}} \xi_{\bar{\nu}+\delta_{p}, \mu}
$$


with $m_{0}=0$. The operator $X_{\mu \nu^{\prime}} z_{n-1, i}$ is transformed in exactly the same manner; cf. [13]. Combining the results we obtain

$$
F_{n-1,-n} \xi_{\nu \mu \nu^{\prime}}=A_{0} \xi(0)+\sum_{i=1}^{n-1} A_{i}\left(\frac{1}{m_{i}} \xi^{+}(i)-\frac{1}{m_{i}-1} \xi^{-}(i)\right)
$$

where

$$
A_{i}=\prod_{a=1, a \neq i}^{n-1} \frac{1}{m_{i}-m_{a}} \prod_{a=1}^{n-1} \frac{1}{m_{i}+m_{a}-1}
$$

Furthermore,

$$
\begin{aligned}
& \xi^{-}(i)=\xi_{\nu, \mu-\delta_{i}, \nu^{\prime}} \\
& \xi^{+}(i)=\sum_{p=1}^{n} \sum_{q=1}^{n-1} \prod_{a=1, a \neq p}^{n} \frac{m_{i}^{2}-\gamma_{a}^{2}}{\gamma_{p}^{2}-\gamma_{a}^{2}} \prod_{a=1, a \neq q}^{n-1} \frac{m_{i}^{2}-\gamma_{a}^{\prime 2}}{{\gamma^{\prime}}^{2}-\gamma_{a}^{\prime 2}} \xi_{\nu+\delta_{p}, \mu+\delta_{i}, \nu^{\prime}+\delta_{q}}
\end{aligned}
$$

and

$$
\begin{array}{rlrl}
\xi(0) & =(-1)^{n} \xi_{\bar{\nu} \mu \bar{\nu}^{\prime}} & \text { if } & \sigma=\sigma^{\prime}=0, \\
& =\sum_{p=1}^{n} \prod_{a=1, a \neq p}^{n} \frac{m_{0}^{2}-\gamma_{a}^{2}}{\gamma_{p}^{2}-\gamma_{a}^{2}} \xi_{\bar{\nu}+\delta_{p}, \mu, \bar{\nu}^{\prime}} & \text { if } \quad \sigma=1, \sigma^{\prime}=0, \\
& =-\sum_{q=1}^{n-1} \prod_{a=1, a \neq q}^{n-1} \frac{m_{0}^{2}-\gamma_{a}^{\prime 2}}{\gamma_{q}^{\prime 2}-\gamma_{a}^{\prime 2}} \xi_{\bar{\nu}, \mu, \bar{\nu}^{\prime}+\delta_{q}} & \text { if } \quad \sigma=0, \sigma^{\prime}=1, \\
& =(-1)^{n-1} \sum_{p=1}^{n} \sum_{q=1}^{n-1} \prod_{a=1, a \neq p}^{n} \frac{m_{0}^{2}-\gamma_{a}^{2}}{\gamma_{p}^{2}-\gamma_{a}^{2}} \prod_{a=1, a \neq q}^{n-1} \frac{m_{0}^{2}-\gamma_{a}^{\prime 2}}{\gamma_{q}^{\prime 2}-\gamma_{a}^{\prime 2} \xi_{\bar{\nu}+\delta_{p}, \mu, \bar{\nu}^{\prime}+\delta_{q}}} \\
& \text { if } \sigma=\sigma^{\prime}=1,
\end{array}
$$

with $\bar{\nu}^{\prime}=\left(\sigma^{\prime}+1, \nu_{1}^{\prime}, \ldots, \nu_{n-1}^{\prime}\right)$ (addition modulo 2$)$. We now compute the action of $F_{n-1, n}$. In the formula (3.9) with $a=n$ replace the operators $z_{i n}$ by with following expression: for $i=-n+1, \ldots, n-1$

$$
z_{i n}=\left[z_{i,-n}, \Phi_{-n, n}\right] \frac{1}{f_{i}+F_{n n}}
$$

and then use the formulas for the action of $z_{i,-n}$ and $\Phi_{-n, n}$; see (3.36). More precisely, we regard (3.38) as a relation in the transvector algebra $\mathrm{Z}\left(\mathfrak{g}_{n}, \mathfrak{g}_{n-1}\right)$ which can be proved as follows. First, we calculate the commutator $\left[F_{i,-n}, \Phi_{-n, n}\right]$ in $\mathrm{U}\left(\mathfrak{g}_{n}\right)$ then consider it modulo the ideal $\mathrm{J}$ and apply the extremal projection $p$ (see Section 2). 
We have $\Phi_{-n, n} F_{n n}=\left(F_{n n}+2\right) \Phi_{-n, n}$ and so, (3.9) and (3.38) imply that

$$
F_{n-1, n} \xi_{\nu \mu \nu^{\prime}}=X_{\mu \nu^{\prime}}\left(\Phi_{n-1,-n}(2) \Phi_{-n, n}-\Phi_{-n, n} \Phi_{n-1,-n}(0)\right) \xi_{\nu \mu}
$$

where

$$
\Phi_{n-1,-n}(u)=\sum_{i=-n+1}^{n-1} z_{n-1, i} z_{i,-n} \prod_{a=-n+1, a \neq i}^{n-1} \frac{1}{f_{i}-f_{a}} \cdot \frac{1}{u+f_{i}+F_{n n}} .
$$

The action of $\Phi_{n-1,-n}(u)$ is found exactly as that of $F_{n-1,-n}$. Formula (3.39) is valid provided the denominators in (3.40) do not vanish. However, since (3.38) holds in the transvector algebra $\mathrm{Z}\left(\mathfrak{g}_{n}, \mathfrak{g}_{n-1}\right)$, the relation (3.39) holds for generic parameters $\nu, \mu$ and $\nu^{\prime}$ which allows one to get explicit formulas for all matrix elements of $F_{n-1, n}$.

Finally, the proof of Theorem 3.1 is completed by rewriting the above formulas for the action of the generators in terms of the parameters $\sigma_{k}, l_{k i}$ and $l_{k i}^{\prime}$ of the patterns $\Lambda$. The parameters $l_{i}, \gamma_{i}, m_{i}$ are replaced by

$$
l_{i} \mapsto l_{k i}+1 / 2, \quad \gamma_{i} \mapsto l_{k i}^{\prime}+1 / 2, \quad m_{i} \mapsto l_{k-1, i}+1 / 2
$$

Remark. There is another way of calculating the matrix elements of $F_{k-1, k}$ based on the formulas for the action of $T_{-n,-n}(u)$ in the basis $\left\{\zeta_{\nu \mu}\right\}$; see [14.

\section{References}

[1] R. M. Asherova, Yu. F. Smirnov, and V. N. Tolstoy, Projection operators for simple Lie groups, Theor. Math. Phys. 8 (1971), 813-825.

[2] V. Chari, A. Pressley, Yangians and R-matrices. L'Enseign. Math. 36 (1990), $267-302$.

[3] R. G. Donnelly, "Explicit constructions of representations of semisimple Lie algebras", PhD Thesis, University of North Carolina at Chapel Hill, 1997.

[4] R. G. Donnelly, Explicit constructions of the fundamental representations of the symplectic Lie algebras. Preprint.

[5] I. M. Gelfand and M. L. Tsetlin, Finite-dimensional representations of the group of unimodular matrices. Dokl. Akad. Nauk SSSR 71 (1950), 825-828 (Russian). English transl. in: I. M. Gelfand, "Collected papers". Vol II, Berlin: SpringerVerlag 1988. 
[6] I. M. Gelfand and M. L. Tsetlin, Finite-dimensional representations of groups of orthogonal matrices. Dokl. Akad. Nauk SSSR 71 (1950), 1017-1020 (Russian). English transl. in: I. M. Gelfand, "Collected papers". Vol II, Berlin: SpringerVerlag 1988.

[7] M. D. Gould, On the matrix elements of the $U(n)$ generators. J. Math. Phys. 22 (1981), 15-22.

[8] M. D. Gould, Wigner coefficients for a semisimple Lie group and the matrix elements of the $O(n)$ generators. J. Math. Phys. 22 (1981), 2376-2388.

[9] R. Howe, T. Umeda, The Capelli identity, the double commutant theorem and multiplicity-free actions. Math. Ann. 290 (1991), 569-619.

[10] I. G. Macdonald, "Symmetric functions and Hall polynomials", 2nd edition, Oxford University Press, Oxford, 1995.

[11] A. Molev, Gelfand-Tsetlin basis for representations of Yangians. Lett. Math. Phys. 30 (1994), 53-60.

[12] A. I. Molev, Finite-dimensional irreducible representations of twisted Yangians. J. Math. Phys. 39 (1998), 5559-5600.

[13] A. I. Molev, A basis for representations of symplectic Lie algebras. Comm. Math. Phys. 201 (1999), 591-618.

[14] A. I. Molev, A weight basis for representations of even orthogonal Lie algebras. In: "Combinatorial methods in representation theory", Proceedings, Kyoto 1998, to appear; math.RT/9902060

[15] A. I. Molev, Yangians and transvector algebras. Preprint SMS 98-30, University of Sydney; math.QA/9811115

[16] A. Molev, M. Nazarov, and G. Olshanski, Yangians and classical Lie algebras. Russian Math. Surveys 51:2 (1996), 205-282.

[17] J. G. Nagel, M. Moshinsky, Operators that lower or raise the irreducible vector spaces of $U_{n-1}$ contained in an irreducible vector space of $U_{n}$. J. Math. Phys. 6 (1965), 682-694. 
[18] M. Nazarov and V. Tarasov, Yangians and Gelfand-Zetlin bases. Publ. RIMS, Kyoto Univ. 30 (1994), 459-478.

[19] M. Nazarov and V. Tarasov, Representations of Yangians with Gelfand-Zetlin bases. J. Reine Angew. Math. 496 (1998), 181-212.

[20] G. I. Olshanski, Twisted Yangians and infinite-dimensional classical Lie algebras. In: P. P. Kulish (ed.), "Quantum Groups", Lecture Notes in Math. 1510, pp. 103-120. Berlin-Heidelberg: Springer 1992.

[21] S. C. Pang and K. T. Hecht, Lowering and raising operators for the orthogonal group in the chain $O(n) \supset O(n-1) \supset \cdots$, and their graphs. J. Math. Phys. 8 (1967), 1233-1251.

[22] V. O. Tarasov, Irreducible monodromy matrices for the R-matrix of the $X X Z$ model and lattice local quantum Hamiltonians. Theor. Math. Phys. 63 (1985), 440-454.

[23] M. K. F. Wong, Representations of the orthogonal group. I. Lowering and raising operators of the orthogonal group and matrix elements of the generators. J. Math. Phys. 8 (1967), 1899-1911.

[24] D. P. Zhelobenko, The classical groups. Spectral analysis of their finitedimensional representations. Russ. Math. Surv. 17 (1962), 1-94.

[25] D. P. Zhelobenko, On Gelfand-Zetlin bases for classical Lie algebras. In: A. A. Kirillov (ed.), "Representations of Lie groups and Lie algebras", pp. 79106. Budapest: Akademiai Kiado 1985.

[26] D. P. Zhelobenko, An introduction to the theory of S-algebras over reductive Lie algebras. In: A. M. Vershik, D. P. Zhelobenko (eds), "Representations of Lie groups and Related Topics", pp. 155-221. Adv. Studies in Contemp. Math. Vol. 7. New York: Gordon and Breach Science Publishers 1990. 